\title{
PET-CT Hybrid Imaging
}

O. Schober and W. Heindel

New York NY: Thieme, 2010, 296 pages, \$149.95

This book is an authorized and revised translation of the German edition (title: PET-CT) written by a multidisciplinary team of 33 experts in radiology and nuclear medicine at the University Hospital of Münster in Germany, except for one who is at Haukerland University Hospital in Norway. The authors explore the combination of high-resolution anatomic images of multislice spiral CT with the specific and sensitive molecular images of PET in a single examination to give readers a full understanding of this evolving technology.

Reimbursement has led to a growing acceptance of PET/ $\mathrm{CT}$ in the clinical setting and its full integration into clinical practice. PET/CT allows accurate localization of functional processes such as metabolic rates, receptor expression, and cell-cell or cell-tissue interaction. The greater diagnostic sensitivity and higher spatial and temporal resolution of advanced scanners have shortened imaging times and thus increased patient throughput. Health insurance companies and employers are searching for ways to improve the efficient use of radiologic imaging techniques. Inappropriate imaging and unnecessary exposure are of great concern.

There are 6 chapters and an appendix. Chapter 1 deals with the basic principles of imaging techniques and interpretation, as well as radiopharmaceuticals and contrast media. Imaging in children and examination protocols are also included. Chapter 2 is 186 pages covering neoplastic imaging of the brain, head and neck, thyroid, lung and pleura, digestive tract (including liver), urinary tract (including prostate), gynecologic system, and musculoskeletal system. Lymphoma, melanoma, unknown primary cancer, and PET/CT for radiotherapy planning are also discussed. Chapters 3, 4, and 5 discuss inflammatory, cardiovascular, and neurodegenerative diseases, respectively. Chapter 6 deals with development and outlook, with positive future prospects and anticipated important developments. The information supplied by multimodal imaging will greatly benefit individual patient management. The appendix contains useful Internet addresses of professional societies and journals. The glossary of frequently used terms, list of common abbreviations, and updated index are also helpful. The 623 illustrations are clear, lavish, and of high quality, but not all lesions are indicated by arrows.
PET-CT Hybrid Imaging covers the essentials of PET/CT fusion technology in one practical, comprehensive volume and presents an evidence-based look at the most modern and important imaging technique in clinical use today, with an emphasis on tumors. The book provides a systematic discussion of patient preparation, recommendations for imaging protocols for specific indications, and specific examination techniques such as slice orientation and positioning.

This book is intended to contribute to the responsible application of PET/CT in routine clinical work. The chapters adhere to a consistent structure and include a brief assessment of alternative imaging such as MRI or CT, listing advantages and limitations. CT, PET, and PET/CT scans appear side by side throughout the text, with explanations of their interpretation. Easy-to-reference text boxes and sidebars present key concepts, pearls, and pitfalls. Detailed summaries at the end of each chapter facilitate rapid review, and carefully selected suggestions are useful for further reading at the end of each section.

PET-CT Hybrid Imaging is well organized, easily readable, and well illustrated for trainees. It is an excellent preparation tool for the special board examinations. It is also ideal for practicing radiologists and nuclear medicine specialists who need to quickly compare and interpret PET/CT images. Many new and promising target-specific radioligands have emerged in the research laboratory and await translation into the clinic. They are likely to further transform the scope of nuclear medicine, with substantial implications for patient care, including diagnosis and evaluation of the disease and risk assessment. They are also likely to enhance monitoring of therapeutic response and tailoring of patient-specific treatment strategies. With that, I highly recommend this book to both imaging specialists and clinicians.

\author{
E. Edmund Kim \\ M.D. Anderson Cancer Center \\ 1515 Holcombe Blvd., Unit 1264 \\ Houston, TX 77030 \\ E-mail: ekim@di.mdacc.tmc.edu
}

